\title{
Polymorphisms of the FAS and FASL genes and risk of breast cancer
}

\author{
WENMIN WANG, ZHONGQIU ZHENG, WENJIE YU, HUI LIN, BINBIN CUI and FEILIN CAO
}

\author{
Department of Surgical Oncology, Taizhou Hospital, Wenzhou Medical College, Linhai, Zhejiang 317000, P.R. China
}

Received September 13, 2011; Accepted December 13, 2011

DOI: $10.3892 / \mathrm{ol} .2011 .541$

\begin{abstract}
FAS and its ligand FASL are crucial in apoptotic cell death. Loss of FAS and gain of aberrant FASL expression are common features of malignant transformation. This study was designed to investigate whether the functional polymorphisms of FAS -1377G/A (rs2234767) and FASL -844T/C (rs763110) affect the risk of developing breast cancer. Genotypes were analyzed by a polymerase chain reaction-restriction fragment length polymorphism assay in 436 breast cancer patients and 496 healthy controls. In this study, as compared to the wild-type homozygote and heterozygote, the distribution of the FAS -1377GG, GA and AA genotypes among breast cancer patients were significantly different from those among healthy controls $(\mathrm{P}=0.011)$, with the AA genotype being more prevalent among patients than the controls $(\mathrm{P}=0.003)$. Similarly, the frequencies of the FASL -844TT, TC and CC genotypes also significantly differed among breast cancer patients and healthy controls $(\mathrm{P}<0.001)$, with the CC genotype being significantly over-represented in breast cancer patients compared with the controls $(\mathrm{P}<0.001)$. In the unconditional logistic regression model following adjustment for age, the subjects carrying the FAS -1377AA genotype had a 1.75 -fold increased risk [95\% confidence interval (CI), 1.13-2.69] for development of breast cancer compared with patients carrying the GG genotype. Similarly, in the recessive model, the FASL -844CC genotype significantly increased the risk of breast cancer with an odds ratio (OR) of $1.92(95 \%$ CI 1.46-2.54) compared with the $T T$ or $T T+T C$ genotypes. Our results suggest that functional polymorphisms in the death pathway genes FAS and FASL significantly contribute to the occurrence of breast cancer.
\end{abstract}

\section{Introduction}

Malignancy formation is not only associated with unlimited proliferation, but is also associated with the suppression of

Correspondence to: Dr Feilin Cao, Department of Surgical Oncology, Taizhou Hospital, Wenzhou Medical College, 150 Ximen Road, Linhai, Zhejiang 317000, P.R. China

E-mail:dr.caofeilin@yahoo.com.cn

Key words: breast cancer, FAS, FASL, risk apoptosis. Apoptosis, a complex process in which cells neatly commit suicide, exerts critical roles in not only the development, but also the homeostasis and normal functioning of adult multi-cellular organisms (1). The over-functioning of apoptosis during development may result in abortion or abnormalities, while failure of inducing apoptosis of DNA-damaged cells may lead to tumor development (2). Accumulating evidence suggests that the acquired ability to resist apoptosis is a common hallmark of various types of malignant diseases, and that the regulatory defects of components of the apoptosis pathway contribute to tumorigenesis, tumor cell invasion and metastasis $(1,2)$.

FAS (also known as Apo-1 or CD95), a potent member of the death receptor family, plays a key role in apoptotic signaling in many cell types (3). FAS interacts with its FAS-ligand (FASL) to trigger the death signal cascade, and subsequently induces apoptotic cell death (4). The interactions between FAS and FASL have been shown to be involved in the establishment of an immune privileged status of the tumor by inducing FAS-mediated apoptosis in tumor-specific lymphocytes (5). Evidence has shown that a number of tumors exhibit downregulation of FAS or loss-of-function conferring resistance to death signals induced by the immune system, as well as an increased expression of FASL-mediated immune privilege, inducing peripheral tolerance to antigens of normal organ environments via the apoptosis of FAS-positive lymphocytes (5-8). Consequently, a decreased expression of FAS and/ or increased expression of FASL may have promoted malignant transformation and progression (9). In addition, the functional mutations in FAS and FASL genes that impair apoptotic signal transduction have been shown to be associated with an increased risk of various types of cancers (10). Thus, the FAS/ FASL system may be significant in cancer initiation, development and progression, and single nucleotide polymorphisms (SNPs), which possess the potential to alter the expression of FAS and/or FASL, have been proposed to be significant in the genetic susceptibility to cancer.

G to A transition at position -1377 (-1377G/A, rs2234767) in the promoter region of FAS has been found to diminish the affinity for binding to transcriptional factor stimulatory protein 1 (Sp1), resulting in a decreased expression of FAS (11). Nevertheless, the FASL gene also has a functional polymorphism in its promoter, a $\mathrm{T}$ to $\mathrm{C}$ change at position -844 (FASL -844T/C, rs763110), which is located in a binding motif for the transcription factor CAAT/enhancer-binding 
Table I. Distribution of selected characteristics.

\begin{tabular}{|c|c|c|c|c|c|}
\hline \multirow[t]{2}{*}{ Variable } & \multicolumn{2}{|c|}{ Patients $(n=436)$} & \multicolumn{2}{|c|}{ Controls $(n=496)$} & \multirow[t]{2}{*}{ P-value } \\
\hline & $\mathrm{n}$ & $\%$ & $\mathrm{n}$ & $\%$ & \\
\hline Age (years) & & & & & 0.536 \\
\hline$\leq 40$ & 23 & 5.3 & 18 & 3.6 & \\
\hline $41-50$ & 50 & 11.5 & 64 & 12.9 & \\
\hline $51-60$ & 132 & 30.3 & 166 & 33.5 & \\
\hline $61-70$ & 160 & 36.7 & 178 & 35.9 & \\
\hline$>70$ & 71 & 16.3 & 70 & 14.1 & \\
\hline \multicolumn{6}{|c|}{ Metastasis at diagnosis } \\
\hline Stage I & 131 & 30.0 & & & \\
\hline Stage II & 162 & 37.2 & & & \\
\hline Stage III & 90 & 20.6 & & & \\
\hline Stage IV & 53 & 12.2 & & & \\
\hline \multicolumn{6}{|c|}{ Histological differentiation } \\
\hline Low & 56 & 12.8 & & & \\
\hline Intermediate & 166 & 38.1 & & & \\
\hline High & 214 & 49.1 & & & \\
\hline
\end{tabular}

${ }^{\text {aTwo-sided }} \chi^{2}$ test.

protein $\beta$ (12). A higher basal expression of FASL is significantly associated with the $-844 \mathrm{C}$ allele compared with the $-844 \mathrm{~T}$ allele (12). The role of these two functional polymorphisms in carcinogenesis has been extensively studied, and have been shown to be associated with an increased risk of various types of cancer $(13,14)$.

Breast cancer is one of the most common fatal malignant tumors worldwide (15). Downregulation of FAS has been observed in certain carcinomas including breast cancer (16), while FASL is occasionally overexpressed in numerous human tumors, including breast cancer $(17,18)$. Therefore, we hypothesized that the functional polymorphisms, FAS -1377G/A and FASL -844T/C, may increase the risk of breast cancer in a Chinese population owing to the reduced expression of FAS and/or increased expression of FASL. To test this hypothesis, we conducted a hospital-based, case-control study of breast cancer in a Chinese population including 436 breast cancer patients and 496 healthy controls.

\section{Materials and methods}

Study population. This study consisted of 436 patients with breast cancer and 496 healthy controls. Patients were recruited between January 2000 and June 2011 at Taizhou Hospital, Wenzhou Medical College (China). All patients with histopathologically confirmed breast cancer were enrolled. Patients with previous cancer, previous chemotherapy or radiotherapy were excluded. Control subjects were cancer-free individuals and were recruited from persons who visited the same hospital for physical examination. The selection criteria for controls included no individual history of cancer and frequency-matching to the cases by age $( \pm 5$ years). The subjects were unrelated, ethnic Han Chinese. At recruitment, informed consent was obtained from each subject and the information on demographic characteristics, such as age was collected. This study was approved by the institutional review board of Taizhou Hospital, Wenzhou Medical College.

Polymorphism analysis. Genomic DNA was extracted from blood samples of all controls and cases collected at recruitment. FAS -1377G/A and FASL -844T/C genotypes were determined using PCR-based restriction fragment length polymorphism (PCR-RFLP) methods as previously described $(19,20)$. A $10 \%$ masked, random sample of subjects was tested twice by various investigators, and the results were concordant for all masked duplicate sets.

Statistical analysis. The Hardy-Weinberg Equilibrium analysis was performed by comparing the observed and expected genotype frequencies in controls using the $\chi^{2}$ test. Pearson's $\chi^{2}$ test was used to estimate the differences in demographic variables and genotype distribution of FAS -1377G/A and FASL -844T/C between patients and controls. The associations between the polymorphisms and risk of breast cancer were estimated using odds ratios (ORs) and their 95\% confidence intervals (CIs), which were calculated by unconditional logistic regression models following adjustment for age. Data were analyzed by SPSS 16.0 software. $\mathrm{P}<0.05$ was considered to indicate a statistically significant difference.

\section{Results}

Subject characteristics. The analysis included 436 breast cancer patients and 496 controls. The distribution of selected characteristics is shown in Table I. No significant differences were found between cases and controls in terms of median age 
Table II Genotype and allele frequencies of FAS and FASL in breast cancer patients, and their contribution to breast cancer risk.

\begin{tabular}{lllll}
\hline Genotype & \multicolumn{2}{c}{ Patients $(\mathrm{n}=436)$} & & \multicolumn{2}{c}{ Controls $(\mathrm{n}=496)$} & $\mathrm{OR}$ \\
\cline { 2 - 3 } & $\mathrm{n}$ & $\mathrm{n}$ & $\%$ \\
\hline
\end{tabular}

\begin{tabular}{|c|c|c|c|c|c|}
\hline \multicolumn{6}{|l|}{$F A S-1377 G / A$} \\
\hline$G G$ & 138 & 36.8 & 197 & 39.7 & 1.00 \\
\hline$G A$ & 171 & 45.6 & 246 & 49.6 & $0.96(0.71-1.30)$ \\
\hline$A A$ & 66 & 17.6 & 53 & 10.7 & $1.75(1.13-2.69)^{\mathrm{b}}$ \\
\hline$G G+G A$ & 309 & 82.4 & 443 & 89.3 & 1.00 \\
\hline$A$ allele frequency & 0.404 & 0.355 & & & \\
\hline \multicolumn{6}{|l|}{$F A S L-844 T / C$} \\
\hline$T T$ & 32 & 8.5 & 48 & 9.7 & 1.00 \\
\hline$T C$ & 120 & 32.0 & 237 & 47.8 & $0.72(0.43-1.19)$ \\
\hline$T T+T C$ & 152 & 59.5 & 285 & 57.5 & 1.00 \\
\hline$C C$ & 268 & 61.5 & 211 & 42.5 & $1.92(1.46-2.54)^{\mathrm{c}}$ \\
\hline$C$ allele frequency & 0.755 & 0.664 & & & \\
\hline
\end{tabular}

${ }^{\mathrm{a}} \mathrm{ORs}$ and $95 \%$ CIs were calculated by the unconditional logistic regression model adjusted for age and other genotypes where appropriate. ${ }^{\mathrm{b}} \mathrm{P}=0.011$, compared with the GG genotype; ${ }^{\mathrm{P}} \mathrm{P}<0.001$, compared with the $T T$ or $T T+T C$ genotypes.

$(\mathrm{P}=0.536)$. Of the 436 patients, $131(30.0 \%)$ had stage I, 162 (37.2\%) had stage II, 90 (20.6\%) had stage III and 53 patients $(12.2 \%)$ had stage IV breast cancer. In terms of histological differentiation, $56(12.8 \%)$ patients had low-differentiated tumors (Grade I), 166 (38.1\%) patients had intermediatedifferentiated tumors (Grade II) and 214 (49.1\%) patients had high-differentiated tumors (Grade III).

Association between FAS and FASL polymorphisms and risk of breast cancer. The allele frequencies and genotype distributions of FAS -1377G/A and FASL -844T/C in breast cancer patients and controls are shown in Table II. The allele frequencies for FAS -1377A and FASL -844C were 0.355 and 0.664 , respectively, in the controls compared with 0.404 and 0.755 , respectively, in patients. The genotype frequencies of FAS $-1377 \mathrm{G} / \mathrm{A}$ and FASL $-844 \mathrm{~T} / \mathrm{C}$ in the healthy controls conformed to the Hardy-Weinberg equilibrium $(\mathrm{P}=0.064$ and $\mathrm{P}=0.112$, respectively). The distribution of the FAS -1377GG, GA and AA genotypes among breast cancer patients were significantly different from those among the controls $\left(\chi^{2}=8.658 ; \mathrm{P}=0.011\right)$, with the AA genotype being more prevalent among the patients than the controls (17.6 versus $10.7 \%$, $\mathrm{P}=0.003)$. Similarly, the frequencies of the FASL -844TT, TC, $\mathrm{CC}$ genotypes also significantly differed between patients and controls $\left(\chi^{2}=25.560 ; \mathrm{P}<0.001\right)$, with the $C C$ genotype being significantly over-represented in breast cancer patients compared to the controls ( 61.5 versus $42.5 \%, \mathrm{P}<0.001)$. In the unconditional logistic regression model following adjustment for age, the subjects carrying the FAS -1377AA genotype had a 1.75-fold increased risk (95\% CI 1.13-2.69) for the development of breast cancer compared to patients with the GG genotype. Similarly, in the recessive model, the FASL -844CC genotype was associated with a significantly increased risk of breast cancer with an OR of 1.92 (95\% CI 1.46-2.54) compared with the $T T$ or $T T+T C$ genotypes.

\section{Discussion}

This study investigated whether genetic polymorphisms in genes for the death receptor, FAS and its ligand, FASL, have an effect on the risk of developing breast cancer in a Chinese population. Our results revealed that the polymorphisms in the promoter regions of FAS -1377G/A and FASL -844T/C have substantial effects on the risk of breast cancer. The FAS -1377AA and FASL -844CC genotypes were significantly associated with an increased risk for developing breast cancer.

There has been accumulating evidence that functional germline polymorphisms of FAS genes are associated with a high risk of cancer (21-24). Recently, two meta-analyses evaluated the relationship between the FAS -1377 G/A polymorphism and cancer risk. Qiu et al analyzed 10,564 cancer cases and 12,075 controls from 17 studies (25). Zhang et al analyzed 11,461 cases and 12,708 controls from 34 case-control studies (14). The results obtained by these authors were consistent in that the FAS -1377AA genotype was associated with a significantly increased cancer risk compared to the $\mathrm{G} / \mathrm{G}$ genotype $(14,25)$. Another meta-analysis of the FASL -844T/C polymorphism in relation to cancer risk was conducted by Zhang et al. These authors reported 11,105 cancer cases and 11,372 controls from 19 published studies and concluded that the FASL -844T variant allele was associated with a significantly reduced cancer risk (13). Consistent with these results, our study demonstrated that the individuals carrying the FAS -1377AA or FASL -844CC genotype were at an increased risk for developing breast cancer, which is in agreement with the biological plausibility. The FAS $-1377 \mathrm{G}$ to A change disrupts the crucial transcriptional activator Sp1 binding site and reduces promoter activity (11), thus a decrease in FAS expression associated with the -1377 genotype is expected. The FASL -844T/C polymorphism is also located in the promoter region of the gene, and the $\mathrm{C}$ allele has been shown to create 
a binding site for the CAAT/enhancer-binding protein $\beta$ transcription factor, resulting in a significantly higher basal FASL expression in luciferase reporter assay peripheral blood fibrocytes (12). Furthermore, a large body of evidence has demonstrated that the downregulated expression of FAS and heightened expression of FASL are common features of malignant transformation and oncogenic events in the evolution of the majority of types of human cancer $(6,9,26)$. According to these lines of evidence, individuals carrying the FAS-1377AA and/or FASL-844 variant genotypes exhibited an aberrant expression of FAS and FASL and presented a higher risk for developing breast cancer. Furthermore, this current result was also consistent with findings of recent studies of other types of cancer, including cervical (24), pancreatic (27) and renal cancer (28).

In conclusion, this study provides the first evidence that polymorphisms in promoter regions of FAS and FASL are significant in the risk of occurrence of breast cancer in a Chinese population. These results confirm the hypothesis that FAS -1377G/A and FASL -844T/C polymorphisms are the susceptible factors for the development of breast cancer, although further independent studies are required to confirm our results.

\section{Acknowledgements}

This project was supported by the Science Research Fund of Taizhou.

\section{References}

1. Fan XQ and Guo YJ: Apoptosis in oncology. Cell Res 11: 1-7, 2001.

2. Evan GI and Vousden KH: Proliferation, cell cycle and apoptosis in cancer. Nature 411: 342-348, 2001.

3. Andera L: Signaling activated by the death receptors of the TNFR family. Biomed Pap Med Fac Univ Palacky Olomouc Czech Repub 153: 173-180, 2009.

4. Kim R, Emi M, Tanabe K, Uchida Y and Toge T: The role of Fas ligand and transforming growth factor $\beta$ in tumor progression: molecular mechanisms of immune privilege via Fas-mediated apoptosis and potential targets for cancer therapy. Cancer 100: 2281-2291, 2004.

5. Reichmann E: The biological role of the Fas/FasL system during tumor formation and progression. Semin Cancer Biol 12: 309-315, 2002.

6. Randhawa SR, Chahine BG,Lowery-Nordberg M, Cotelingam JD and Casillas AM: Underexpression and overexpression of Fas and Fas ligand: a double-edged sword. Ann Allergy Asthma Immunol 104: 286-292, 2010.

7. Whiteside TL: The role of death receptor ligands in shaping tumor microenvironment. Immunol Invest 36: 25-46, 2007.

8. Fulda S and Pervaiz S: Apoptosis signaling in cancer stem cells. Int J Biochem Cell Biol 42: 31-38, 2010

9. Houston A and O'Connell J: The Fas signalling pathway and its role in the pathogenesis of cancer. Curr Opin Pharmacol 4: 321-326, 2004.

10. Wajant H: The Fas signaling pathway: more than a paradigm. Science 296: 1635-1636, 2002.

11. Huang QR, Morris D and Manolios N: Identification and characterization of polymorphisms in the promoter region of the human Apo-1/Fas (CD95) gene. Mol Immunol 34: 577-582, 1997.
12. Wu J, Metz C, Xu X, Abe R, Gibson AW, Edberg JC, Cooke J, Xie F, Cooper GS and Kimberly RP: A novel polymorphic CAAT/enhancer-binding protein $\beta$ element in the FasL gene promoter alters Fas ligand expression: a candidate background gene in African American systemic lupus erythematosus patients. J Immunol 170: 132-138, 2003.

13. Zhang Z, Qiu L, Wang M, Tong N, Li J and Zhang Z: The FAS ligand promoter polymorphism, rs763110 (-844C $>\mathrm{T})$, contributes to cancer susceptibility: evidence from 19 case-control studies. Eur J Hum Genet 17: 1294-1303, 2009.

14. Zhang Z, Xue H, Gong W, Wang M, Yuan L, Han S and Zhang Z: FAS promoter polymorphisms and cancer risk: a meta-analysis based on 34 case-control studies. Carcinogenesis 30: 487-493, 2009.

15. Siegel R, Ward E, Brawley O and Jemal A: Cancer statistics, 2011: the impact of eliminating socioeconomic and racial disparities on premature cancer deaths. CA Cancer J Clin 61: 212-236, 2011.

16. Keane MM, Ettenberg SA, Lowrey GA, Russell EK and Lipkowitz S: Fas expression and function in normal and malignant breast cell lines. Cancer Res 56: 4791-4798, 1996.

17. Herrnring C, Reimer T, Jeschke U, Makovitzky J, Krüger K, Gerber B, Kabelitz D and Friese K: Expression of the apoptosis-inducing ligands FasL and TRAIL in malignant and benign human breast tumors. Histochem Cell Biol 113: 189-194, 2000.

18. Müllauer L, Mosberger I, Grusch M, Rudas M and Chott A: Fas ligand is expressed in normal breast epithelial cells and is frequently up-regulated in breast cancer. J Pathol 190: 20-30, 2000.

19. Sun T, Miao X, Zhang X, Tan W, Xiong P and Lin D: Polymorphisms of death pathway genes FAS and FASL in esophageal squamous-cell carcinoma. J Natl Cancer Inst 96: 1030-1036, 2004.

20. Zhang X, Miao X, Sun T, Tan W, Qu S, Xiong P, Zhou Y and Lin D: Functional polymorphisms in cell death pathway genes FAS and FASL contribute to risk of lung cancer. J Med Genet 42: 479-484, 2005.

21. Lai HC, Sytwu HK, Sun CA, Yu MH, Yu CP, Liu HS, Chang CC and Chu TY: Single nucleotide polymorphism at Fas promoter is associated with cervical carcinogenesis. Int J Cancer 103: 221-225, 2003.

22. Liu Y, Wen QJ, Yin Y, Lu XT, Pu SH, Tian HP, Lou YF, Tang YN Jiang X, Lu GS and Zhang J: FASLG polymorphism is associated with cancer risk. Eur J Cancer 45: 2574-2578, 2009.

23. Wang LH, Ting SC, Chen $\mathrm{CH}$, Tsai CC, Lung O, Liu TC, Lee CW, Wang YY, Tsai CL and Lin YC: Polymorphisms in the apoptosis-associated genes FAS and FASL and risk of oral cancer and malignant potential of oral premalignant lesions in a Taiwanese population. J Oral Pathol Med 39: 155-161, 2010.

24. Kang S, Dong SM, Seo SS, Kim JW and Park SY: FAS -1377 G/A polymorphism and the risk of lymph node metastasis in cervical cancer. Cancer Genet Cytogenet 180: 1-5, 2008.

25. Qiu LX, Shi J, Yuan H, Jiang X, Xue K, Pan HF, Li J and Zheng MH: FAS $-1,377 \mathrm{G} / \mathrm{A}$ polymorphism is associated with cancer susceptibility: evidence from 10,564 cases and 12,075 controls. Hum Genet 125: 431-435, 2009.

26. Shimonishi T, Isse K, Shibata F, Aburatani I, Tsuneyama K, Sabit H, Harada K, Miyazaki K and Nakanuma Y: Up-regulation of fas ligand at early stages and down-regulation of Fas at progressed stages of intrahepatic cholangiocarcinoma reflect evasion from immune surveillance. Hepatology 32: 761-769, 2000.

27. Yang M, Sun T, Wang L, Yu D, Zhang X, Miao X, Liu J, Zhao D, Li H, Tan W and Lin D: Functional variants in cell death pathway genes and risk of pancreatic cancer. Clin Cancer Res 14: 3230-3236, 2008.

28. Zhu J, Qin C, Wang M, Yan F, Ju X, Meng X, Ding Q, Li P, Yang J, Cao Q, Zhang Z and Yin C: Functional polymorphisms in cell death pathway genes and risk of renal cell carcinoma. Mol Carcinog 49: 810-817, 2010. 